\title{
Morphological and Rheological Properties of Starches Separated from Cultivars of Rice (Oryza sativa L.) from North East India
}

\author{
Nikhil K. Chrungoo*, Ayam Gangarani Devi \\ Depatment of Botany, North Eastern Hill University, Shillong, India \\ Email: nchrungoo@gmail.com
}

Received 26 June 2015; accepted 22 August 2015; published 25 August 2015

Copyright (C) 2015 by authors and Scientific Research Publishing Inc.

This work is licensed under the Creative Commons Attribution International License (CC BY). http://creativecommons.org/licenses/by/4.0/

(c) (i) Open Access

\section{Abstract}

Starch granules isolated from different accessions of rice from North East India showed a typical polygonal morphology with size ranging from $3.4 \pm 0.8 \mu \mathrm{m}$ to $6.4 \pm 1.2 \mu \mathrm{m}$. The apparent amylose content (AAC) of the granules varied from $1.9 \%$ to $28.33 \%$. Our results identify starch from IC583088 as "waxy" and IC-583085, IC-583038, DPRR-168 as "very low" amylo-. The coefficient of resistance to flow (n) for starch pastes from the varieties of rice studied in the present investigation recorded a value of $<1.0$. The observed " $n$ " value deviates from the Newtonian flow indicating the pseudoplastic nature of starches isolated from these varieties. While starch pastes from the accession IC-583088 recorded the highest " $n$ " value of 0.6 , that from the accession IC-545197 showed the lowest value of 0.03 . The variations in coefficient of resistance to flow clearly revealed a higher " $n$ " value for starch pastes from the "waxy" and "low" amylo-cultivars than the "intermediate" or "high" amylo-cultivars. Our results clearly established characteristic rheological properties for starches from the accessions SKY-AK-1608, IC-583035, YS-RC-219, IC-564939 and IC-332963, which exhibited greater resistance to thinning and shearing than other varieties.

\section{Keywords}

Rice, Starch, Apparent Amylose Content, Rheology

\section{Introduction}

Starch, an insoluble polymer that occurs in plant cells in the form of a complex granular mixture of amylose and amylopectin, is one of the major dietary sources of carbohydrates and the most abundant storage polysaccharide in plants. Starch is synthesized in amyloplasts in the form of granules with a partially crystalline texture. De-

\footnotetext{
"Corresponding author.
}

How to cite this paper: Chrungoo, N.K. and Devi, A.G. (2015) Morphological and Rheological Properties of Starches Separated from Cultivars of Rice (Oryza sativa L.) from North East India. American Journal of Plant Sciences, 6, $2019-2031$. 
pending on the number of starch granules initiated in each amyloplast, they have been classified as simple, compound and semi-compound [1]. Compound starch granules are composed of several sub-granules, each of which is synthesized simultaneously within a single amyloplast. Depending upon the species and the tissue, the granules vary in size from 1 to $100 \mu \mathrm{m}$ and in shape from round to polygonal [2]. Starch granules of wheat, barley, rye and triticale have been reported to show bimodal size distribution in the endosperm tissues and have been classified into two types viz., " $\mathrm{A}$ " type which is lenticular in shape with diameter ranging between 10 and $20 \mu \mathrm{m}$ and "B" type which is spherical in shape with a diameter of $<10 \mu \mathrm{m}$ [3]-[7]. Starches of rice and oat tend to exist in clusters of individual granules designated as compound granules [8]-[11]. " $\mathrm{C}$ " type starches are considered to be a mixture of A- and B-type polymorphs. While the "A" and "C" type starches are characteristic of cereals and legumes, respectively, the "B" type structures are characteristics of starches from roots and tuber tissues [12]-[14].

The functional properties of starches from different sources vary considerably and are therefore unique for each type. These differences arise from variations in amylose to amylopectin ratio [15] [16], presence of lipids [17] and in morphology [18]. According to Tester and Morrison [19] while starch swelling is a property associated with amylopectin, amylose in starch restricts it [15] [16]. Thus, while low amylose flour is good for preparation of oriental noodles as well as for extended shelf life of bread, flour with high amylose content is good for fried foods [20] [21].

As the primary dietary source of carbohydrates for human nutrition, rice plays an important role in meeting energy requirements and nutrient intake [22]. Juliano [8] has highlighted the unique characteristics of rice starch and has emphasized that it can be a better substitute for many other different types of starches including that from corn. North East India, being at the confluence of Indo-Malayan and Indo-Chinese biogeographic realms, covering an area of $2.55 \times 10^{5} \mathrm{~km}^{2}$ from $21^{\circ} 57^{\prime}$ to $29^{\circ} 28^{\prime} \mathrm{N}$ latitude and from $89^{\circ} 40^{\prime}$ to $97^{\circ} 25^{\prime} \mathrm{E}$ longitude, represents one of the "biodiversity hotspots" of the world. Even though the region houses a large diversity of both upland and lowland cultivars of rice which show large variations in some of the key quality attributes including kernel length, aroma and cooking quality [23], there is no information on the quality characteristics of starches from cultivars of rice from this region. The paper reports the physico-chemical and rheological properties of starch from cultivars of rice from North East India differing in their amylose-amylopectin ratio.

\section{Materials and Methods}

\subsection{Plant Material}

Grains of different accessions of rice (Oryza sativa L.) from North East India, representing cultivars with varying apparent amylose content (AAC) of starch, were procured from the rice germplasm bank of North Eastern Regional Station of National Bureau of Plant Genetic Resources, India. The collection comprised of 24 upland and 20lowland accessions from the region. The collection was maintained ex situ in the experimental field of the University. Grains of each accession were raised to seedling stage in $6 \mathrm{~cm}$ deep plastic trays filled with 50:50 mixture of soil and compost which was irrigated with water to cover the soil surface. The seedlings were subsequently transferred to 2 meter $\times 2$ meter low level beds raised for the purpose in the experimental fields of the University and maintained ex situ under irrigation till maturity at an average day temperature ranging between $19^{\circ} \mathrm{C}$ to $22^{\circ} \mathrm{C}$. Transplanting of seedlings was done at a spacing of $20 \times 15 \mathrm{~cm}$ with the soil submerged in 1 to 2 $\mathrm{cm}$ of water up to 10 days after transplanting. The seedlings were dipped in chloropyriphos: water (1:1000) solution before transplanting for preventing insect attack. Fertilizers were applied @ 80:60:40 kg NPK/ha in split dozes with $\mathrm{P}, \mathrm{K}$ and $50 \%$ of $\mathrm{N}$ applied as the basal dose, $25 \% \mathrm{~N}$ at maximum tillering and rest $25 \% \mathrm{~N}$ at panicle initiation stage. Grains were harvested at maturity from plants of each accession maintained in the experimental field.

\subsection{Isolation of Starch Granules}

Starch granules were isolated from mature grains of rice according to Takaoka et al. [24]. The granules were homogenized to fine powder under liquid nitrogen in a chilled pestle and mortar. The powder was subsequently suspended in deionised water and the suspension maintained at $4^{\circ} \mathrm{C}$ for 4 hours with occasional stirring. The suspension was filtered through a single layer of muslin and the filtrate centrifuged at $10,000 \times \mathrm{g}$ at $4^{\circ} \mathrm{C}$ for 10 min. The pellet was resuspended in appropriate volume of SDS sample buffer containing $60 \mathrm{mM}$ Tris- $\mathrm{HCl}(\mathrm{pH}$ 
6.8), $10 \%(\mathrm{w} / \mathrm{v})$ sodium dodecylsulfate (SDS), $3 \%(\mathrm{v} / \mathrm{v}) \beta$-mercaptoethanol and $10 \%(\mathrm{v} / \mathrm{v})$ glycerol and then pelleted by centrifugation at $30,000 \mathrm{~g}$ at $4^{\circ} \mathrm{C}$ for $10 \mathrm{~min}$. The resultant pellet was resuspended again in SDS sample buffer, pelleted by centrifugation at $30,000 \times \mathrm{g}$ at $4^{\circ} \mathrm{C}$ for $10 \mathrm{~min}$ and finally washed twice with deionised water followed by three washes with chilled acetone. The washed pellet comprising of purified starch granules was dried at room-temperature and stored at $-20^{\circ} \mathrm{C}$.

\subsection{Scanning Electron Microscopy of Isolated Starch Granules}

Surface morphology of the starch granules was determined under a scanning electron microscope after spraying a thin layer of purified starch granules on 'brass stubs' and coating with a thin layer of gold vapour ( $300 \AA)$ as per David et al. [25]. The starch granules were subsequently observed in a JOEL-1850 SEM at magnification ranging between $7500 \times$ and $10000 \times$ operated at $20 \mathrm{kV}$.

\subsection{Determination of Apparent Amylose Content}

The apparent amylose content of starch was determined by the method described by Juliano [26]. A suitable mass of starch was suspended in an appropriate volume of $95 \%$ ethanol: $1 \mathrm{~N} \mathrm{NaOH} \mathrm{(1:9).} \mathrm{The} \mathrm{suspension} \mathrm{was}$ mixed thoroughly and heated at $100^{\circ} \mathrm{C}$ for $10 \mathrm{~min}$. in a dry bath. A suitable aliquot of the suspension was mixed with $1 \mathrm{~N}$ acetic acid and $\mathrm{I}_{2}-\mathrm{KI}$ solution and allowed to stand for $20 \mathrm{~min}$. in dark at room temperature. Absorbance of the solution was recorded at $620 \mathrm{~nm}$ in Lambda650 UV/Vis Spectrophotometer (Perkin Elmer, U.S.A.) against a reagent blank with potato amylose (Sigma) as the reference standard. Starch isolated from grains of var. NDR97, a traditional variety of indigenous "Bororice" which is known to have low (4\%) AAC [27], was used as a reference for purposes of comparison.

\subsection{Determination of Rheological Properties}

The rheologicalproperties of starches suspended in distilled water were determined by Rotational rheometer (C-DG26.7/QC, RheolabQC) according to Li et al. [28]. An 8\% (w/v) suspension of native rice starch was prepared by dispersing a suitable mass of dried starch granules in appropriate volume of MilliQ deionized water with constant stirring. After loading the starch suspension between the cone and plate, it was equilibrated to $50^{\circ} \mathrm{C}$ for $1 \mathrm{~min}$ at $200 \mathrm{~s}^{-1}$ followed by increase in the temperature to $95^{\circ} \mathrm{C}$ at the rate of $12^{\circ} \mathrm{C} \cdot \mathrm{min}^{-1}$. The sample was held at $95^{\circ} \mathrm{C}$ for $2 \mathrm{~min}$. and 30 seconds after which the temperature was decreased to $50^{\circ} \mathrm{C}$ at the rate of $12^{\circ} \mathrm{C} \cdot \mathrm{min}^{-1}$. The sample was finally held at $50^{\circ} \mathrm{C}$ for $1 \mathrm{~min}$. The shear rate was maintained at $200 \mathrm{~s}^{-1}$ throughout the experiment. The pasting properties including onset temperature, peak temperature, peak viscosity, trough viscosity, final viscosity, breakdown viscosity and setback viscosity of each sample were inferred from acquired data.

\subsection{Statistical Analysis}

All the observations were recorded with 3 technical and 3 biological replicates. Statistical analysis of the data was carried out with SPSS package to obtain the values for standard deviation from the mean as well as least significant difference between accessions for grain size, AAC, and pasting properties of starch at $5 \%$ probability level.

\section{Results and Discussion}

The accessions showed an average plant height of $110-152 \mathrm{~cm}$. While the upland accessions showed an average maturity time of 105 days, the lowland accessions showed an average maturity time of 123 days from the date of transplanting. Grains of different accessions of rice (Oryza sativa L.), representing both upland and lowland varieties from North East India, varied in length from $7.75 \mathrm{~mm}$ to $3.5 \mathrm{~mm}$ and in width from $2.05 \mathrm{~mm}$ to $3.1 \mathrm{~mm}$ (Table 1 and Table 2). While the grains of accession IC-583088 showed the highest length to width ratio (L/W) of 3.6, those from IC-540182 and SKY-AK-1608 showed the lowest L/W of 1.2. Cruz and Khush [29] have classified rice grains into different size and shape categories on the basis of their morphology. While grains with a length of $>7.5 \mathrm{~mm}$ were classified as very long, those with average length between 6.61 and $7.5 \mathrm{~mm}$ and between 5.51 and $6.6 \mathrm{~mm}$ were respectively classified as long and intermediate type. Grains with a length of $<$ or $=$ 
Table 1. Morphological features of grains of different upland accessions of rice from Northeast region of India.

\begin{tabular}{|c|c|c|c|c|c|c|}
\hline Rice variety & $\begin{array}{l}\text { Mean grain } \\
\text { length }^{\dagger}(\mathrm{mm})\end{array}$ & $\begin{array}{l}\text { Mean grain } \\
\text { width }^{\dagger}(\mathbf{m m})\end{array}$ & $\begin{array}{c}\text { Grain length/width } \\
\text { ratio }^{* * \dagger}\end{array}$ & Grain type $^{\mathrm{a}}$ & $\begin{array}{l}\text { Mean grain } \\
\text { weight }^{\dagger}(g)\end{array}$ & Grain colour \\
\hline IC-583088 & $7.75 \pm 0.57(a)$ & $2.15 \pm 0.12(e)$ & $3.6 \pm 0.28(g)$ & LS & $0.022 \pm 0.005(i)$ & White \\
\hline IC-583085 & $6.6 \pm 0.42(b)$ & $2.05 \pm 0.09(e)$ & $3.2 \pm 0.3(g)$ & MS & $0.018 \pm 0.002(i)$ & Light cream \\
\hline DPRR-168 & $5.5 \pm 0.46(\mathrm{c})$ & $3.1 \pm 0.09(f)$ & $1.8 \pm 0.15(h)$ & SB & $0.020 \pm 0.003(i)$ & Light green \\
\hline IC-583038 & $4.5 \pm 0.30(c)$ & $3.1 \pm 0.18(f)$ & $1.5 \pm 0.1(h)$ & SB & $0.024 \pm 0.004(i)$ & Light green \\
\hline SKY-AK-1608 & $3.87 \pm 0.48(d)$ & $3.1 \pm 0.2(f)$ & $1.2 \pm 0.09(h)$ & SB & $0.018 \pm 0.002(i)$ & Off white \\
\hline IC-583035 & $4.12 \pm 0.57(c)$ & $3.1 \pm 0.2(f)$ & $1.3 \pm 0.09(h)$ & SB & $0.026 \pm 0.004(i)$ & Light green \\
\hline YS-RC-219 & $5.5 \pm 0.40(c)$ & $3.1 \pm 0.16(f)$ & $1.8 \pm 0.09(h)$ & SB & $0.020 \pm 0.003(i)$ & Light brown \\
\hline VP-28 & $5.0 \pm 0.62(c)$ & $3.1 \pm 0.2(f)$ & $1.6 \pm 0.087(h)$ & SB & $0.017 \pm 0.003(i)$ & Brown \\
\hline SKY-AK-1569 & $6.65 \pm 0.58(b)$ & $2.15 \pm 0.14(e)$ & $3.0 \pm 0.18(g)$ & MM & $0.026 \pm 0.005(i)$ & Off white \\
\hline IC-540276 & $3.87 \pm 0.82(d)$ & $2.15 \pm 0.16(e)$ & $1.8 \pm 0.17(h)$ & SB & $0.017 \pm 0.005(i)$ & Light brown \\
\hline YS-RC-27 & $5.5 \pm 0.81(c)$ & $3.1 \pm 0.21(f)$ & $1.8 \pm 0.15(h)$ & SB & $0.024 \pm 0.004(i)$ & Off white \\
\hline IC-526602 & $6.0 \pm 0.79(c)$ & $3.1 \pm 0.21(f)$ & $1.9 \pm 0.155(h)$ & MB & $0.020 \pm 0.003(i)$ & Brown \\
\hline IC-398030 & $6.25 \pm 0.89(c)$ & $3.1 \pm 0.19(f)$ & $2.0 \pm 0.16(h)$ & MB & $0.031 \pm 0.005(i)$ & Brown \\
\hline IC-583129 & $6.7 \pm 0.91(c)$ & $2.6 \pm 0.20(e)$ & $2.6 \pm 0.18(g)$ & LM & $0.020 \pm 0.005(i)$ & Off white \\
\hline IC-583032 & $5.7 \pm 0.77(c)$ & $2.15 \pm 0.16(e)$ & $2.6 \pm 0.18(g)$ & MM & $0.016 \pm 0.005(i)$ & Light green \\
\hline IC-540182 & $3.75 \pm 0.61(d)$ & $3.1 \pm 0.22(f)$ & $1.2 \pm 0.11(h)$ & SB & $0.015 \pm 0.005(i)$ & Light green \\
\hline VP-15 & $4.87 \pm 0.65(c)$ & $3.1 \pm 0.22(f)$ & $1.6 \pm 0.14(h)$ & SB & $0.017 \pm 0.006(i)$ & Brown \\
\hline SKY-AK-1556 & $5.0 \pm 0.61(c)$ & $2.15 \pm 0.19(e)$ & $2.3 \pm 0.20(g)$ & SM & $0.026 \pm 0.005(i)$ & Light green \\
\hline SKY-AK-1549 & $6.75 \pm 0.71(c)$ & $3.1 \pm 0.20(f)$ & $2.2 \pm 0.20(g)$ & LM & $0.022 \pm 0.007(i)$ & Off white \\
\hline IC-583029 & $6.12 \pm 0.70(c)$ & $2.15 \pm 0.15(e)$ & $2.8 \pm 0.25(g)$ & MM & $0.022 \pm 0.007(i)$ & Brown \\
\hline YS-RC-105 & $5.0 \pm 0.78(c)$ & $2.15 \pm 0.15(e)$ & $2.3 \pm 0.2(g)$ & SM & $0.021 \pm 0.007(i)$ & Brown \\
\hline IC-583113 & $6.0 \pm 0.88(c)$ & $3.1 \pm 0.21(f)$ & $1.7 \pm 0.1(h)$ & MB & $0.018 \pm 0.006(i)$ & Off white \\
\hline VP-44 & $7.2 \pm 0.71(c)$ & $3.34 \pm 0.18(f)$ & $3.3 \pm 0.12(g)$ & LS & $0.026 \pm 0.008(i)$ & Brown \\
\hline IC-540270 & $7.5 \pm 0.77(c)$ & $2.15 \pm 0.13(e)$ & $3.48 \pm 0.27(g)$ & LS & $0.026 \pm 0.008(i)$ & Off white \\
\hline
\end{tabular}

${ }^{{ }^{*} \mathrm{~S}} \mathrm{SB}$ (short-bold), LM (long-medium), LS (long-slender), MS (medium-slender), MM (medium-medium), SM (short-medium), MB (medium-bold) (as per Dela Cruz and Khush [29]); 'Same letters in parenthesis against data for each parameter indicates statistically insignificant difference $(p<$ $0.05)$.

Table 2. Morphological features of grains of different lowland accessions of rice from Northeast region of India.

\begin{tabular}{|c|c|c|c|c|c|c|}
\hline Rice variety & $\begin{array}{c}\text { Mean grain } \\
\text { length }^{\dagger}(\mathbf{m m})\end{array}$ & $\begin{array}{l}\text { Mean grain } \\
\text { width }^{\dagger}(\mathbf{m m})\end{array}$ & $\begin{array}{c}\text { Grain length/width } \\
\text { ratio }^{*_{\dagger}}\end{array}$ & Grain Type $^{a}$ & $\begin{array}{l}\text { Mean grain } \\
\text { weight }^{\dagger}(g)\end{array}$ & Grain colour \\
\hline IC-564948 & $7.0 \pm 0.11(a)$ & $2.15 \pm 0.04(e)$ & $3.2 \pm 0.13(g)$ & LS & $0.022 \pm 0.002(i)$ & Reddish brown \\
\hline IC-564953 & $6.6 \pm 0.32(b)$ & $2.15 \pm 0.04(e)$ & $3.0 \pm 0.14(g)$ & MM & $0.021 \pm 0.002(i)$ & Light green \\
\hline IC-564950 & $5.0 \pm 0.21(b)$ & $2.15 \pm 0.04(e)$ & $2.3 \pm 0.10(g)$ & SM & $0.018 \pm 0.001(i)$ & Light green \\
\hline IC-321204 & $6.25 \pm 0.25(c)$ & $2.15 \pm 0.03(e)$ & $2.9 \pm 0.10(g)$ & MM & $0.021 \pm 0.002(i)$ & Reddish brown \\
\hline IC-558321 & $5.0 \pm 0.12(b)$ & $3.1 \pm 0.06(f)$ & $1.6 \pm 0.12(h)$ & SB & $0.021 \pm 0.002(i)$ & Light yellow \\
\hline IC-321201 & $6.75 \pm 0.42(c)$ & $2.15 \pm 0.03(e)$ & $3.1 \pm 0.15(g)$ & LS & $0.022 \pm 0.003(i)$ & Light yellow \\
\hline IC-564939 & $6.5 \pm 0.34(c)$ & $3.1 \pm 0.05(f)$ & $2.0 \pm 0.08(h)$ & MB & $0.020 \pm 0.003(i)$ & Light yellow \\
\hline IC-332963 & $6.0 \pm 0.43(c)$ & $2.15 \pm 0.04(e)$ & $2.7 \pm 0.17(g)$ & MM & $0.022 \pm 0.002(i)$ & Reddish brown \\
\hline IC-324133 & $6.37 \pm 0.24(c)$ & $2.15 \pm 0.04(e)$ & $2.9 \pm 0.20(g)$ & MM & $0.022 \pm 0.002(i)$ & Reddish brown \\
\hline IC-321195 & $5.0 \pm 0.17(b)$ & $2.15 \pm 0.05(e)$ & $2.3 \pm 0.11(g)$ & SM & $0.019 \pm 0.005(i)$ & Reddish brown \\
\hline IC-558311 & $5.75 \pm 0.17(b)$ & $2.15 \pm 0.04(e)$ & $2.6 \pm 0.11(g)$ & MM & $0.025 \pm 0.005(i)$ & Light yellow \\
\hline IC-545288 & $3.5 \pm 0.08(d)$ & $2.15 \pm 0.04(e)$ & $1.6 \pm 0.11(h)$ & SB & $0.019 \pm 0.002(i)$ & Light green \\
\hline IC-564949 & $5.75 \pm 0.09(b)$ & $2.15 \pm 0.05(e)$ & $2.6 \pm 0.16(g)$ & MM & $0.016 \pm 0.002(i)$ & Light green \\
\hline IC-321206 & $3.87 \pm 0.09(d)$ & $2.15 \pm 0.04(e)$ & $1.8 \pm 0.09(h)$ & SB & $0.019 \pm 0.002(i)$ & Reddish brown \\
\hline IC-564952 & $5.0 \pm 0.10(b)$ & $3.1 \pm 0.06(f)$ & $1.6 \pm 0.10(h)$ & SB & $0.020 \pm 0.003(i)$ & Greenish yellow \\
\hline IC-321193 & $6.12 \pm 0.19(c)$ & $2.15 \pm 0.04(e)$ & $2.8 \pm 0.14(g)$ & MM & $0.023 \pm 0.005(i)$ & Reddish brown \\
\hline IC-564940 & $5.37 \pm 0.17(b)$ & $2.15 \pm 0.05(e)$ & $2.4 \pm 0.13(g)$ & SM & $0.023 \pm 0.005(i)$ & Light yellow \\
\hline IC-545274 & $4.25 \pm 0.19(d)$ & $3.1 \pm 0.07(f)$ & $1.3 \pm 0.06(h)$ & SB & $0.020 \pm 0.004(i)$ & Greenish yellow \\
\hline IC-558315 & $7.0 \pm 0.25(a)$ & $2.15 \pm 0.05(e)$ & $3.2 \pm 0.12(g)$ & LS & $0.020 \pm 0.004(i)$ & Greenish yellow \\
\hline IC-545197 & $6.75 \pm 0.30(a)$ & $2.15 \pm 0.05(e)$ & $3.1 \pm 0.11(g)$ & LS & $0.022 \pm 0.005(i)$ & Reddish brown \\
\hline
\end{tabular}

${ }^{{ }^{*} \mathrm{a}} \mathrm{SB}$ (short-bold), LM (long-medium), LS (long-slender), MS (medium-slender), MM (medium-medium), SM (short-medium), MB (medium-bold) (as per Dela Cruz and Khush [29]); ${ }^{\dagger}$ Same letters in parenthesis against data for each parameter indicates statistically insignificant difference $(p<$ 0.05 ). 
$5.5 \mathrm{~mm}$ were classified as short type. Further, Cruz and Khush [29] classified grains with L/W of >3.0, 2.1 to 3.0 and $<2.0$ as slender, medium and bold respectively. In conformity with this classification, rice grains from accessions studied in the present investigation can be classified into seven categories viz., long-slender (LS), longmedium (LM), medium-slender (MS), medium-medium (MM), medium-bold (MB), short-medium (SM) and short-bold (SB). The accessions also showed marked variations in the colour of pericarp (Table 1 and Table 2).

Starch granules isolated from different accessions, representing both upland and lowland cultivars of rice from North East India, showed a typical polygonal morphology (Figure 1 and Figure 2) with a mono-modal distribution and size ranging from a minimum of $3.4 \pm 0.8 \mu \mathrm{m}$ in IC-564948 to a maximum of $6.7 \pm 1.2 \mu \mathrm{m}$ in IC-540270 (Table 3). A distinct feature of starch granules isolated from accessions YS-RC-105, IC-321201 and IC-332963 was the presence of pores on the surface of the granules. No such pores were visible on the surface of

Table 3. Size and apparent amylose content of starch granules isolated from endosperm tissues of grains of upland and lowland accessions of rice from North East India. 'Same letters in parenthesis against data for each parameter indicates statistically insignificant difference $(p<0.05)$.

\begin{tabular}{|c|c|c|c|c|c|c|c|}
\hline Accessions & $\begin{array}{c}\text { Granule size } \\
(\mu \mathrm{m})\end{array}$ & $\begin{array}{c}\mathrm{AAC}^{\dagger} \\
(\%)\end{array}$ & Type & Accessions & $\begin{array}{c}\text { Granule size } \\
(\mu \mathrm{m})\end{array}$ & $\begin{array}{c}\mathrm{AAC}^{\dagger} \\
(\%)\end{array}$ & Type \\
\hline \multicolumn{4}{|c|}{ Upland } & \multicolumn{4}{|c|}{ Lowland } \\
\hline IC-583088 & $3.7 \pm 0.8(a)$ & $1.90 \pm 0.08(b)$ & Waxy & IC-564948 & $3.4 \pm 0.8(a)$ & $13.78 \pm 0.57(f)$ & Low amylo \\
\hline IC-583085 & $3.6 \pm 1.1(a)$ & $4.15 \pm 0.25(c)$ & Very low amylo & IC-564953 & $3.5 \pm 0.5(a)$ & $14.10 \pm 0.72(f)$ & Low amylo \\
\hline DPRR-168 & $3.9 \pm 1.0(a)$ & $10.27 \pm 0.52(d)$ & Very low amylo & IC-558321 & $4.1 \pm 0.7(a)$ & $14.94 \pm 0.70(f)$ & Low amylo \\
\hline IC-583038 & $4.1 \pm 0.8(a)$ & $11.80 \pm 0.48(e)$ & Very low amylo & IC-564939 & $4.2 \pm 0.8(a)$ & $15.14 \pm 0.69(f)$ & Low amylo \\
\hline IC-540276 & $4.5 \pm 0.8(a)$ & $15.74 \pm 0.78(\mathrm{f})$ & Low amylo & IC-558311 & $4.7 \pm 0.6(a)$ & $18.73 \pm 1.30(h)$ & Low amylo \\
\hline IC-564950 & $3.9 \pm 1.0(a)$ & $14.3 \pm 1.26(f)$ & Low amylo & IC-321204 & $4.0 \pm 1.1(a)$ & $14.72 \pm 1.6(f)$ & Low amylo \\
\hline SKY-AK-1608 & $4.0 \pm 0.9(a)$ & $14.0 \pm 1.38(f)$ & Low amylo & IC-545288 & $4.9 \pm 0.5(a)$ & $19.63 \pm 1.73(h)$ & Low amylo \\
\hline YS-RC-219 & $4.2 \pm 0.6(a)$ & $14.21 \pm 1.38(f)$ & Low amylo & IC-321201 & $4.2 \pm 1.2(a)$ & $15.11 \pm 1.86(f)$ & Low amylo \\
\hline IC-583035 & $4.2 \pm 0.6(a)$ & $14.12 \pm 1.58(f)$ & Low amylo & IC-332963 & $4.3 \pm 1.9(a)$ & $15.25 \pm 1.16(f)$ & Low amylo \\
\hline VP-28 & $4.2 \pm 1.3(a)$ & $14.35 \pm 1.57(f)$ & Low amylo & IC-324133 & $4.4 \pm 1.5(a)$ & $16.24 \pm 1.89(f)$ & Low amylo \\
\hline SKY-AK-1569 & $4.3 \pm 0.7(a)$ & $14.82 \pm 1.28(f)$ & Low amylo & IC-321195 & $4.6 \pm 0.4(a)$ & $17.52 \pm 1.23(f)$ & Low amylo \\
\hline YS-RC-27 & $4.5 \pm 1.1(a)$ & $16.59 \pm 0.95(g)$ & Low amylo & IC-321206 & $4.8 \pm 1.7(a)$ & $21.8 \pm 0.0(h)$ & Intermediate amylo \\
\hline IC-398030 & $4.8 \pm 1.0(a)$ & $19.61 \pm 1.03(h)$ & Low amylo & IC-564949 & $4.9 \pm 1.1(a)$ & $21.62 \pm 0.0(h)$ & Intermediate amylo \\
\hline IC-526602 & $4.4 \pm 1.6(a)$ & $19.5 \pm 1.85(h)$ & Low amylo & IC-545274 & $5.3 \pm 0.7(a)$ & $25.52 \pm 1.51(k)$ & High amylo \\
\hline IC-583129 & $4.8 \pm 0.6(a)$ & $20.08 \pm 0.91(h)$ & Low amylo & IC-558315 & $5.7 \pm 0.8(a)$ & $26.65 \pm 1.53(k)$ & High amylo \\
\hline IC-583032 & $4.7 \pm 1.1(a)$ & $21.62 \pm 1.02(h) \mathrm{I}$ & Intermediate amylo & IC-545197 & $5.8 \pm 1.2(a)$ & $27.85 \pm 1.76(k)$ & High amylo \\
\hline IC-540182 & $4.9 \pm 0.5(a)$ & $21.80 \pm 0.99(h) \mathrm{I}$ & Intermediate amylo & IC-564952 & $5.0 \pm 0.1(a)$ & $25.22 \pm 1.23(k)$ & High amylo \\
\hline VP-15 & $4.9 \pm 0.7(a)$ & $21.90 \pm 1.11(h) \mathrm{I}_{1}$ & Intermediate amylo & IC-321193 & $5.1 \pm 0.4(a)$ & $25.40 \pm 1.35(k)$ & High amylo \\
\hline SKY-AK-1556 & $4.9 \pm 1.9(a)$ & $21.90 \pm 0.93(h) \mathrm{I}$ & Intermediate amylo & IC-564940 & $5.1 \pm 1.1(a)$ & $25.41 \pm 1.31(k)$ & High amylo \\
\hline SKY-AK-1549 & $5.0 \pm 0.1(a)$ & $21.90 \pm 1.31(h) \mathrm{I}$ & ntermediate amylo & & & & \\
\hline YS-RC-105 & $5.3 \pm 0.8(a)$ & $23.72 \pm 1.52(j) \mathrm{I}$ & ntermediate amylo & & & & \\
\hline IC-583029 & $5.2 \pm 0.6(a)$ & $26.32 \pm 1.58(k)$ & High amylo & & & & \\
\hline IC-583113 & $5.2 \pm 1.9(a)$ & $26.7 \pm 1.88(k)$ & High amylo & & & & \\
\hline VP-44 & $5.5 \pm 0.2(a)$ & $28.33 \pm 1.88(k)$ & High amylo & & & & \\
\hline IC-540270 & $6.4 \pm 1.2(a)$ & $28.33 \pm 1.97(k)$ & High amylo & & & & \\
\hline
\end{tabular}




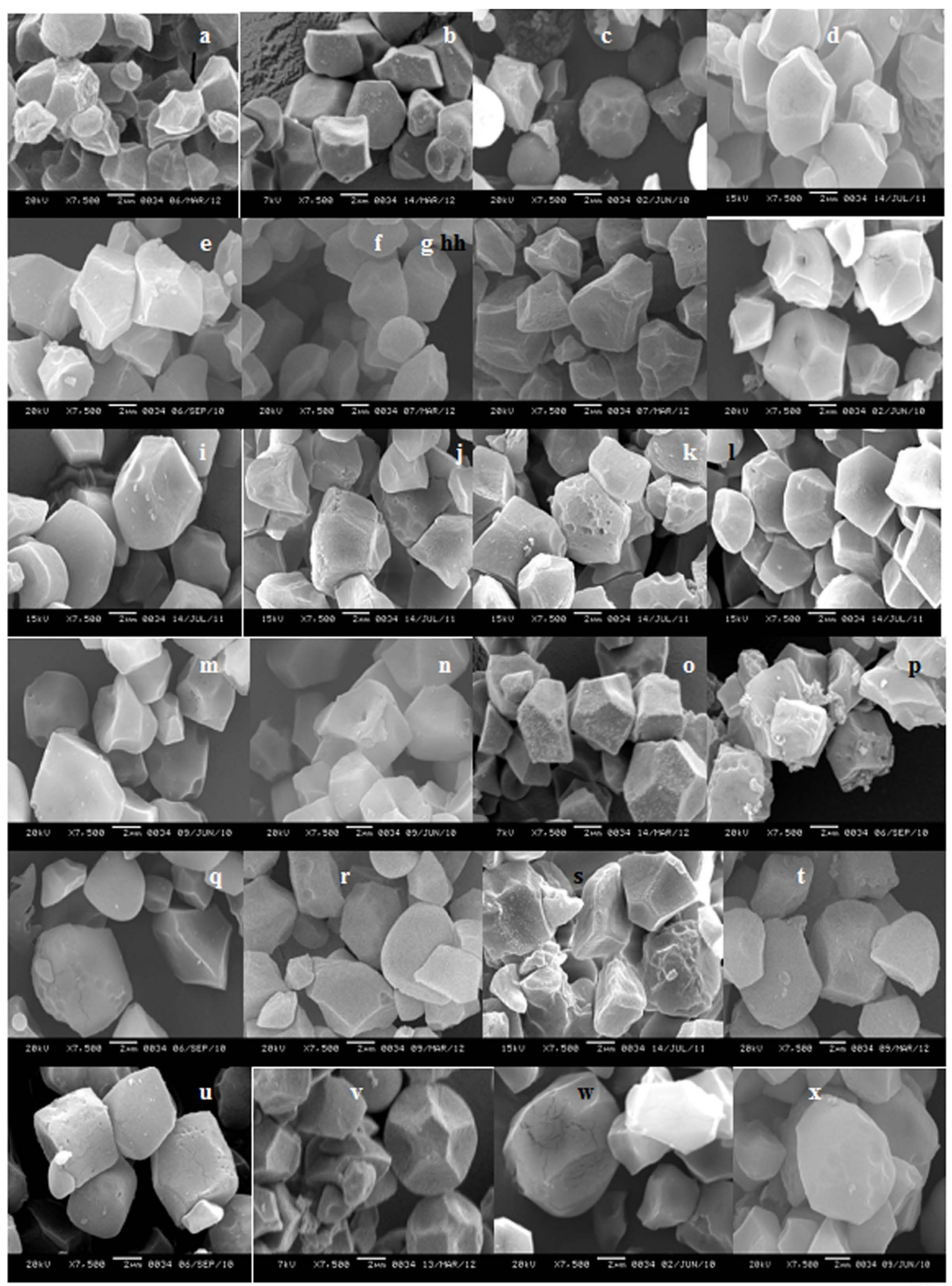

Figure 1. (a) Scanning electron micrographs of starch granules isolated from upland varieties of rice from Northeast India. (a) IC-583088; (b) IC-583085; (c) DPRR-168; (d) IC-583038; (e) SKY-AK-1608; (f) IC-583035; (g) YS-RC-219; (h) VP-28; (i) SKY-AK-1569; (j) IC-540276; (k) YS-RC-27; (1) IC-526602; (m) IC-398030; (n) IC-583129; (o) IC-583032; (p) IC-540182, (q) VP-15; (r) SKY-AK-1556; (s) SKY-AK-1549; (t) IC-583029; (u) YS-RC-105; (v) IC-583113; (w) VP-44; (x) IC-540270. 

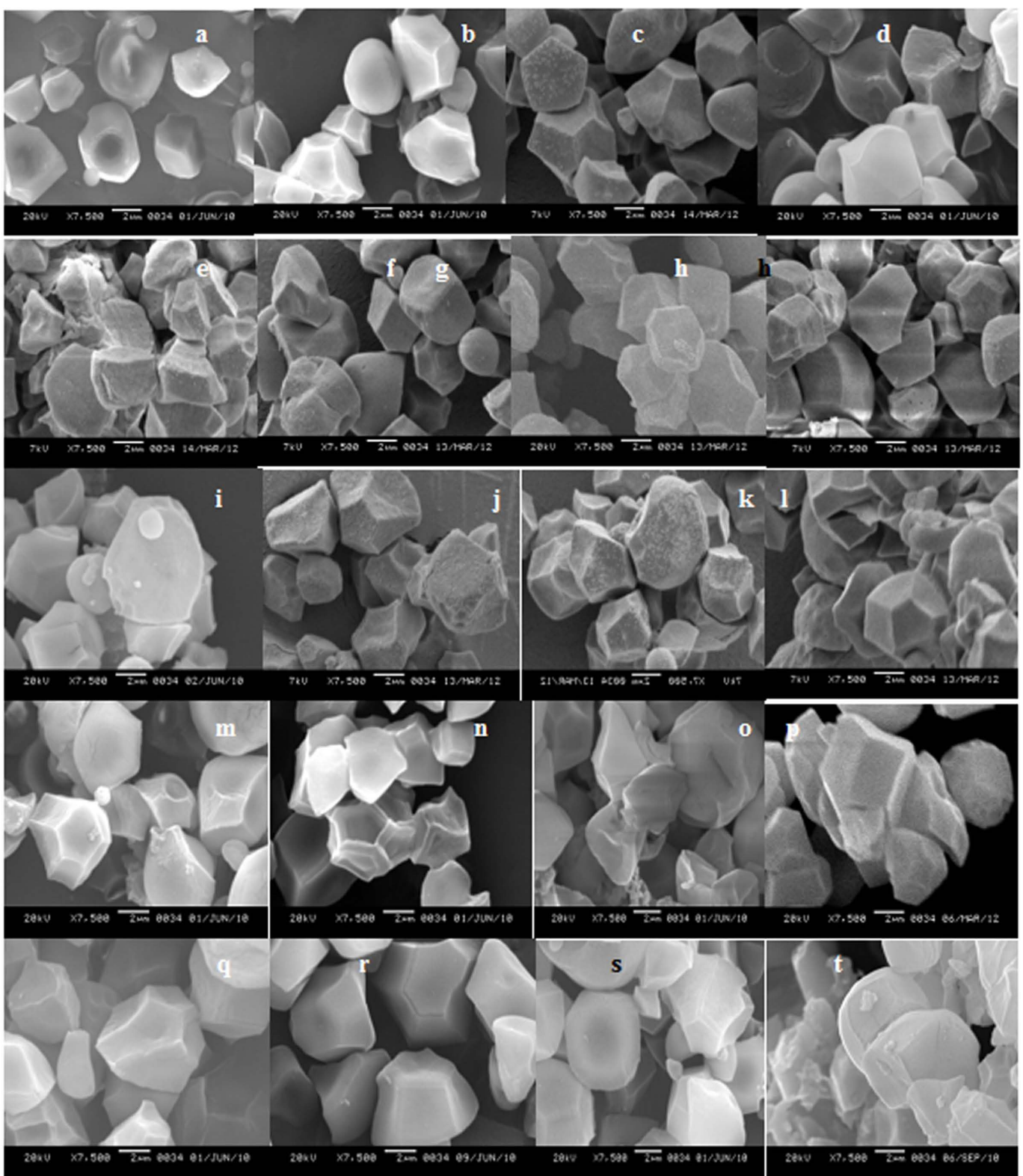

Figure 2. Scanning electron micrographs of starch granules isolated from lowland varieties of rice from Northeast India (a) IC-564948; (b) IC-564953; (c) IC-564950; (d) IC-321204; (e) IC-558321; (f) IC-321201; (g) IC-564939; (h) IC-332963; (i) IC-324133; (j) IC-321195, (k) IC-558311; (l) IC-545288; (m) IC-564949; (n) IC-321206; (o) IC-564952; (p) IC-321193; (q) IC-964940; (r) IC-545274; (s) IC-558315; (t) IC-545197.

starch granules from other accessions. On the basis of their size, Lindeboom et al. [7] have classified starch granules as large $(>25.0 \mu \mathrm{m})$, medium $(10.0$ to $25.0 \mu \mathrm{m})$, small $(5.0$ to $10.0 \mu \mathrm{m})$ and very small $(<5.0 \mu \mathrm{m})$. In conformity with this classification, starch granules isolated the accessions of rice investigated in the present study fall under the "small" to "very small" size category. Juliano [8], Yeh and Li [30] and Li and Yeh [31] have also reported the presence of small starch granules in rice with size ranging between $2.0 \mu \mathrm{m}$ and $10.0 \mu \mathrm{m}$. Starch granule size has been described as an important parameter which has strong correlation with its pasting behaviour [32]-[34].

The apparent amylose content (AAC) of starch from different accessions of rice studied in the present investigation ranged from a low of $1.9 \%$ in IC-583088 to a high of $28.33 \%$ in IC-540270 and VP-44 (Table 3). Even though typical levels of amylose in starches range from $15 \%$ to $25 \%$ [34], the amylose content is known to vary 
with source of the starch and is affected by the climatic conditions as well as the nutrient status of soil during grain development. Our results on AAC of starch in different accessions of rice are in agreement with Wang et al. [36] who have reported $18.1 \%$ to $31.6 \%$ AAC in starches of different non-glutinous cultivars and $0.1 \%$ to $3.25 \%$ AAC in starches of different glutinous cultivars of rice. While Dobo et al. [37] have classified non-glutinous starches into high $(>25 \%)$, intermediate $(21 \%-24 \%)$ and low $(15 \%-20 \%)$ AAC types, Jiminez et al. [38] have suggested categorization of non-glutinous starches into high (>25\%), intermediate $(20 \%-25 \%)$ and low $(<20 \%)$ AAC types. Considering the broad range of amylose content in the low AAC category, starches with $<20 \%$ AAC have been further categorized into "waxy" (0\% - 2\%), "very low" amylo- $(5 \%-12 \%)$ and "low" amylo- (12\% - 20\%) types [39]-[41]. In conformity with this classification, the starch of IC-583088 can be classified as "waxy" and that of IC-583085, IC-583038 and DPRR-168 as "very low" amylo-types. While the starches from IC-540276, YS-RC-27, IC-398030, IC-583129, IC-564948, IC-564953, IC-558321, IC-564939 and IC558311 can be classified as "low" amylo-, that from IC-583032, IC-540182, VP-15, SKY-AK-1556, SKY-AK1549, YS-RC-105 and from IC-564952, IC-321193, IC-564940, IC-545274, IC-583029, IC-558315, IC-545197, VP-44 and IC-540270 can be respectively grouped under the "intermediate" and "high" amylo-types. Jayamani et al. [42] have made similar classifications for a collection of International germplasm maintained in Portugese rice germplasm bank.

Marked differences were observed in the rheological properties of starches isolated from different accessions of rice studied in the present investigation. While the values for shear stress recorded a steady increase with increase in shear rate, the magnitude of increase varied from accession to accession. Thus, starch from the accession IC-583088 showed the lowest of 3.6 fold increase in shear stress and that from VP-44 showed the highest of 119 fold increase in shear stress with increase in shear rate from 0.054 to $100 \mathrm{~s}^{-1}$ (Table 4 and Table 5). The

Table 4. Relationship between shear stress and shear rates of $4 \%(\mathrm{w} / \mathrm{v})$ aqueous pastes of starches from upland varieties of rice from Northeast India.

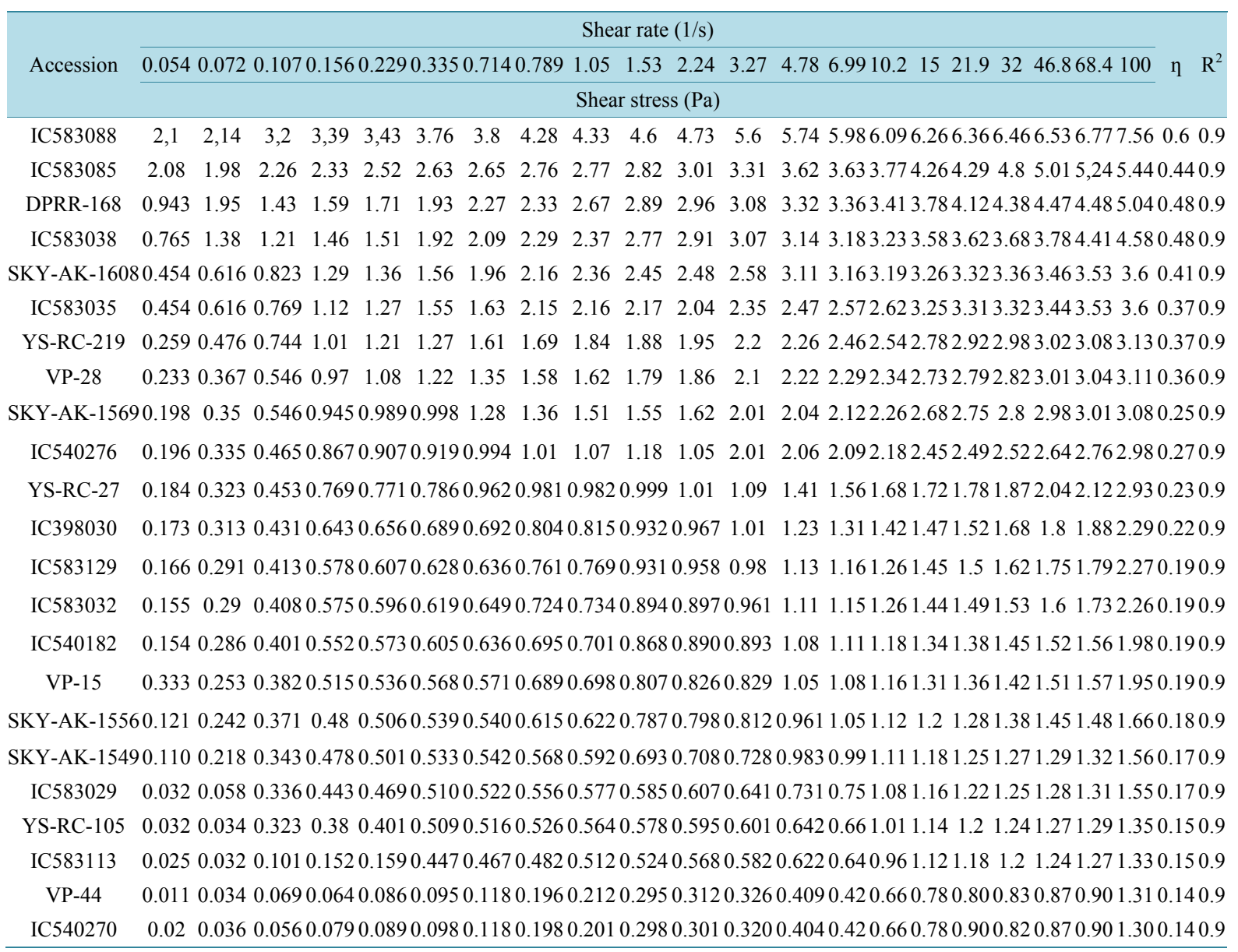


Table 5. Relationship between shear stress and shear rates of $4 \%(\mathrm{w} / \mathrm{v})$ aqueous pastes of starches from lowland varieties of rice from Northeast India.

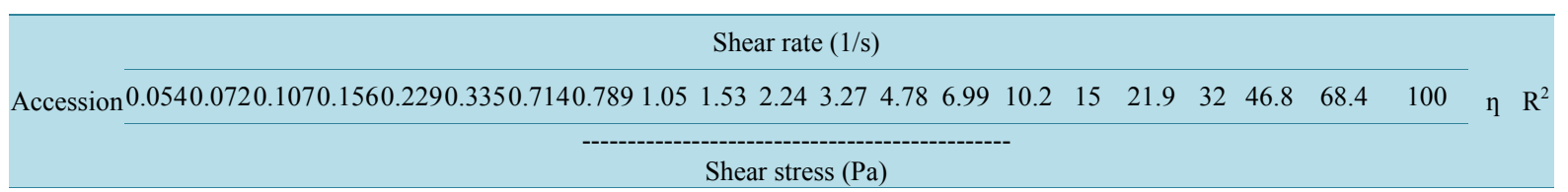

IC564948 0.1720.3670.5670.867 $1.35 \quad 1.68 \quad 2.16 \quad 2.56 \quad 2.84 \quad 2.96 \quad 3.08 \quad 3.14 \quad 3.18 \quad 3.23 \quad 3.26 \quad 3.31 \quad 3.36 \quad 3.44 \quad 3.53 \quad 3.6 \quad 3.65 \quad 0.450 .9$



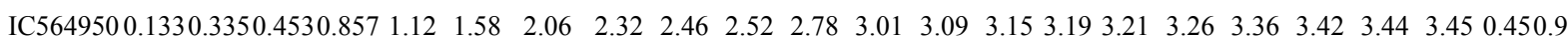

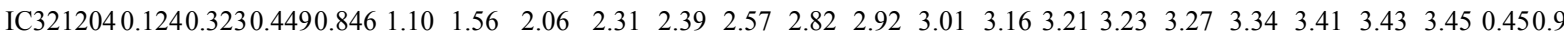

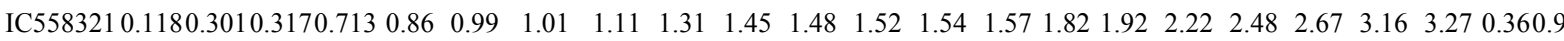

$\begin{array}{llllllllllllllllllllllll}\text { IC321201 } & 0.11 & 0.2560 .3050 .665 & 0.83 & 0.88 & 0.91 & 1.13 & 1.17 & 1.33 & 1.31 & 1.39 & 1.41 & 1.66 & 1.69 & 1.88 & 2.14 & 2.34 & 2.56 & 2.85 & 3.16 & 0.340 .9\end{array}$

$\begin{array}{llllllllllllllllllllll}\text { IC564939 } & 0.11 & 0.2380 .299 & 0.64 & 0.68 & 0.87 & 0.92 & 0.99 & 1.05 & 1.19 & 1.22 & 1.31 & 1.34 & 1.56 & 1.66 & 1.74 & 2.06 & 2.33 & 2.44 & 2.67 & 3.12 & 0.330 .9\end{array}$

$\begin{array}{lllllllllllllllllllllll}\text { IC332963 } & 0.10 & 0.2320 .2760 .5150 .536 & 0.63 & 0.66 & 0.79 & 0.90 & 1.03 & 1.11 & 1.21 & 1.32 & 1.40 & 1.60 & 1.68 & 1.94 & 2.22 & 2.42 & 2.46 & 2.85 & 0.330 .9\end{array}$

$\begin{array}{lllllllllllllllllllll}\text { IC324133 } & 0.09 & 0.23 & 0.2560 .4970 .5180 .568 & 0.64 & 0.7240 .8040 .894 & 0.95 & 1.10 & 1.23 & 1.34 & 1.50 & 1.62 & 1.72 & 1.87 & 2.19 & 2.32 & 2.46 & 0.290 .9\end{array}$

IC321195 0.0780.2180.2280.4150.5010.539 $0.61 \quad 0.66 \quad 0.7300 .8680 .9430 .996 \quad 1.08 \quad 1.19 \quad 1.45 \quad 1.50 \quad 1.64 \quad 1.89 \quad 2.12 \quad 2.25 \quad 2.29 \quad 0.280 .9$

$\begin{array}{llllllllllllllllllllll}\text { IC558311 } & 0.06 & 0.2160 .225 & 0.41 & 0.51 & 0.533 & 0.612 & 0.6560 .7340 .8580 .943 & 1.09 & 1.10 & 1.20 & 1.40 & 1.40 & 1.62 & 1.84 & 2.04 & 2.28 & 2.29 & 0.280 .9\end{array}$

IC564949 0.0180.1720.1910.3680.4800.498 $0.571 \quad 0.6150 .7270 .8320 .935 \quad 0.98 \quad 1.05 \quad 1.08 \quad 1.12 \quad 1.14 \quad 1.18 \quad 1.25 \quad 1.28 \quad 1.31 \quad 1.32 \quad 0.170 .9$

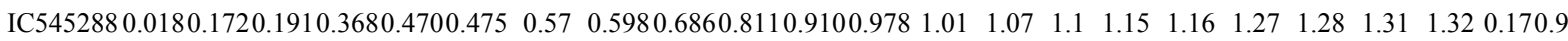



IC564952 0.0110.1110.1750.2990.4060.447 0.467 0.505 0.5630.5780.5850.6050.6420.661 0.660.6840.7050.744 0.77 0.7850.8460.090.9

IC321193 0.011 $0.10 \quad 0.1660 .2520 .3050 .3250 .3470 .3600 .4040 .4140 .4330 .4550 .4730 .5100 .520 .5270 .5430 .5670 .5860 .7430 .7970 .070 .9$

IC564940 0.0110.0580.1280.1520.2190.228 0.234 0.262 0.2810.2950.3430.3640.4040.4170.430.4410.4510.4690.4900.5420.6650.060.9

IC545274 0.0090.0920.1160.1210.1590.195 0.196 0.2120.2590.289 0.31 0.3270.341 0.3430.350.3750.4130.4570.4730.5610.5740.060.9

IC558315 0.0090.0550.0990.1160.153 $0.19 \quad 0.196 \quad 0.2010 .2350 .286 \quad 0.310 .3270 .3390 .3370 .340 .373 \quad 0.410 .4560 .4730 .5230 .5730 .060 .9$

IC545197 0.0060.0340.0660.0790.0860.088 0.091 0.0990.1050.1190.1220.1390.1410.1660.17 0.21 0.2130.2220.2420.2460.3120.030.9

coefficient of resistance to flow (n) for the starch pastes recorded a value of $<1.0$, with starch paste from IC583088 showing the highest value of 0.6 and that from IC-545197 showing the lowest value of 0.03 . The observed " $n$ " value deviates from the Newtonian flow, for which $n=1$, thereby indicating the pseudoplastic nature of the starches isolated from these varieties. The non-Newtonian behaviour of starch pastes has also been reported by Rao et al. [43] and Nurul et al. [44]. Our results on the variations in coefficient of resistance to flow (n) of starch pastes from different accessions of rice clearly revealed a higher " $n$ " value for starch pastes from the "waxy" and "low amylo"-cultivars than the "intermediate" or "high" amylo-cultivars, thereby indicating a positive relationship between amylose/amylopectin ratio of starch and its flow behaviour. Starch pastes from all the accessions of rice studied in the present investigation showed the shear-thinning phenomenon. This is evident from a decrease in apparent viscosity of the starch pastes with increase in shear rate, thereby increasing its fluidity. Our results on the variations in shear stresses clearly revealed that starch pastes from the "waxy" and "low" amylo-cultivars exhibited higher shear stress than the "intermediate" or "high" amylo-cultivars at the same shear rate. This indicates a clear relationship between the amylose/amylopectin ratio of starch grains and their rheological properties. Since higher values for shear stress indicate higher structural stability of the starch granules [45], it can be assumed that "waxy" and "low" amylo-starches have a more stable structure than those with intermediate or high AAC.

Marked variations were observed in the pasting properties of the starches from accessions of rice studied in the present investigation. While the pasting onset temperatures of the "waxy" and "very low" amylo-cultivars were within the range of $61.6^{\circ} \mathrm{C}-62.9^{\circ} \mathrm{C}$, those for the "low" and "intermediate" amylo-cultivars were in the range of $63.47^{\circ} \mathrm{C}-66.36^{\circ} \mathrm{C}$ and $67.26^{\circ} \mathrm{C}-73.52^{\circ} \mathrm{C}$, respectively (Table 6 and Table 7). Our results on the past- 
Table 6. Pasting properties of starch isolated from upland varieties of rice from Northeast India. \pm represents standard deviation, ${ }^{\dagger}$ Same letters in parenthesis against data for each parameter indicates statistically insignificant difference $(p<0.05)$.

\begin{tabular}{|c|c|c|c|c|c|c|c|}
\hline Rice variety & $\begin{array}{c}\text { Onset Temp }{ }^{\dagger} \\
\left({ }^{\circ} \mathrm{C}\right)\end{array}$ & $\begin{array}{c}\text { Peak Temp }{ }^{\dagger} \\
\left({ }^{\circ} \mathbf{C}\right)\end{array}$ & $\begin{array}{l}\text { Peak Visco }{ }^{\dagger} \\
\text { (Pa.s) }\end{array}$ & $\begin{array}{c}\text { Trough Visco } \\
\text { (Pa.s) }\end{array}$ & $\begin{array}{c}\text { Final Visco }{ }^{\dagger} \\
\text { (Pa.s) }\end{array}$ & $\begin{array}{c}\text { Breakdown } \\
\text { Visco }^{\dagger} \text { (Pa.s) }\end{array}$ & $\begin{array}{l}\text { Setback } \\
\text { viscosity }\end{array}$ \\
\hline IO & $(a)$ & (a) & $\pm 0.0(j)$ & i) & $0.44 \pm 0.0(b)$ & $0.345 \pm 0.0(a)$ & $25 \pm 0.0(a)$ \\
\hline IC-58 & $(f)$ & a) & $0(i j)$ & $0.435=$ & $0.30 \pm 0.0(a)$ & $0.39 \pm 0$ & \\
\hline DPRF & (c) & $0(a)$ & $.0(i)$ & 0.43 & $0.32=$ & 0.405 & $0(k)$ \\
\hline $1 C-583038$ & $.6 \pm 0.1(b)$ & $.11 \pm 0.0(a)$ & $0.81 \pm 0.0(i)$ & $0.44 \pm 0.0(h)$ & $0.56 \pm 0.0(d)$ & $0.41 \pm 0.0(b)$ & $0.28 \pm 0.0(k)$ \\
\hline SKY-AK-1608 & $63.47 \pm 0.0(d)$ & $2 \pm 0.0(b)$ & $0.65 \pm 0.0(h)$ & $0.43 \pm 0.0(g)$ & $0.82 \pm 0.0(i j)$ & & \\
\hline IC-583035 & $64.44 \pm$ & $82.26 \pm 0.0(c)$ & $0.66 \pm 0.0(h)$ & $0.42 \pm 0.0(f g)$ & $0.835 \pm 0.0(j)$ & $0.25 \pm 0.0(d e)$ & $0.51=$ \\
\hline YS-RC & & $84.34 \pm$ & $0.62 \pm 0.0(g)$ & $0.435 \pm 0.0(h)$ & $0.835 \pm$ & & \\
\hline VP-28 & $64.49 \pm 0.0(f)$ & $83.53 \pm 0.0(d)$ & $0.61 \pm 0.0(g)$ & $0.415 \pm 0.0(e)$ & $0.62 \pm 0.0(e)$ & $0.30 \pm 0.0(f)$ & $0.0(c d)$ \\
\hline SKY-AK-1 & $65.59 \pm 0.0(g)$ & $86.19 \pm 0.2(e)$ & $0.61 \pm$ & $0.41 \pm 0$. & $0.64 \pm 0$ & $0.265 \pm 0.0(c d)$ & $0.28=$ \\
\hline & & & & & & & \\
\hline YS-R & $63.49 \pm$ & $87.10 \pm$ & $0.58 \pm 0.0(g)$ & $0.36 \pm 0.0(\mathrm{~d} e f)$ & $0.52 \pm 0.0(c)$ & $0.18=$ & 0.28 \\
\hline IC-52 & $66.20 \pm 0$ & $88.47 \pm 0.3(g)$ & $0.48 \pm 0.0(f)$ & $0.365 \pm 0.0($ def $)$ & $0.67 \pm 0.0(g h)$ & $.0(i)$ & $0.30 \pm$ \\
\hline IC-398030 & $65.83 \pm 0.0(i)$ & $89.89 \pm 0.0(i j)$ & $0.43 \pm 0.0(e)$ & $0.37 \pm 0.0(f g)$ & $0.68 \pm 0.0(h)$ & $0.09 \pm 0.0(j)$ & $0.30 \pm 0.0(c)$ \\
\hline IC-583129 & $66.36 \pm 0.0(h)$ & $88.34 \pm 0.0(g)$ & $.042 \pm 0.0(e)$ & $0.37 \pm 0.0(d e)$ & $0.79 \pm 0.0(i)$ & $0.16 \pm 0.0(k)$ & $0.30 \pm 0.0(c)$ \\
\hline IC-583032 & $67.26 \pm 0.0(j)$ & $88.88 \pm 0.0(g h)$ & $0.39 \pm 0.0(c d)$ & $0.36 \pm 0.0(c d e)$ & $0.875 \pm 0.0(k l)$ & $0.145 \pm 0.0(k)$ & $0.30 \pm 0.0(c d)$ \\
\hline IC-540182 & $71.16=$ & $89.53 \pm 0.0(h i)$ & $0.38 \pm 0.0 \odot$ & $0.36 \pm 0.0(c d e)$ & $0.865 \pm 0.0(k)$ & $0.135 \pm 0.0(i)$ & $0.44 \pm 0.0(e)$ \\
\hline VP-15 & $73.25 \pm 0.0(n)$ & $90.33 \pm 0.0(i j)$ & $0.36 \pm 0.0(b c)$ & $0.34 \pm 0.0(c d)$ & $0.835 \pm 0.0(j)$ & $0.11 \pm 0.0(m)$ & $0.43 \pm 0.0(e)$ \\
\hline SKY-AK-1556 & $72.81 \pm 0.0(\mathrm{~m})$ & $90.56 \pm 0.2(j k)$ & $0.37 \pm 0.0(b c)$ & $0.30 \pm 0.0(b c)$ & $0.82 \pm 0.0(i j)$ & $0.105 \pm 0.0(\mathrm{~m})$ & $0.46 \pm 0.0(g)$ \\
\hline SKY-AK-1549 & $73.5 \pm 0.0(n)$ & $91.29 \pm 0.3(k l)$ & $0.37 \pm 0.0(b c)$ & $0.24 \pm 0.0(b)$ & $0.895 \pm 0.0(\mathrm{~m})$ & $0.105 \pm 0.0(\mathrm{~m})$ & $0.45 \pm 0.0(f g)$ \\
\hline IC-583029 & $73.52 \pm 0.0(n)$ & $91.5 \pm 0.4(l)$ & $0.36 \pm 0.0(c)$ & $0.26 \pm 0.0(b)$ & $0.885 \pm 0.0(l)$ & $0.105 \pm 0.0(m)$ & $0.43 \pm 0.0(e)$ \\
\hline YS-RC-105 & $72.05 \pm 0.0(l)$ & $92.6 \pm 0.0(1 \mathrm{~m})$ & $0.33 \pm 0.0(b)$ & $0.27 \pm 0.0(b)$ & $0.90 \pm 0.0(\mathrm{~m})$ & $0.05 \pm 0.0(n)$ & $0.51 \pm 0.0(h)$ \\
\hline IC-583113 & $74.33 \pm 0.0(o)$ & $92.5 \pm 0.0(\mathrm{~m})$ & $0.21 \pm 0.0(a)$ & $0.17 \pm 0.0(a)$ & $0.91 \pm 0,0(m)$ & $0.03 \pm 0.0(f)$ & $0.545 \pm 0.0(j)$ \\
\hline VP-44 & $75.58 \pm 0.0(p)$ & $92.12 \pm 0.0(\mathrm{~lm})$ & $0.21 \pm 0.0(a)$ & $0.18 \pm 0.0(a)$ & $0.965 \pm 0.0(n)$ & $0.027 \pm 0.0(c)$ & $0.52 \pm 0.0(i)$ \\
\hline IC-540270 & $75.82 \pm 0.0(p)$ & $92.78 \pm 0.0(m)$ & $0.22 \pm 0.0(a)$ & $0.18 \pm 0.0(a)$ & $0.98 \pm 0.0(n)$ & $0.027 \pm 0.0(c)$ & $0.52 \pm 0.0(h i)$ \\
\hline
\end{tabular}

Table 7. Pasting properties of starch isolated from lowland varieties of rice from Northeast India. \pm represents standard deviation, ${ }^{\dagger}$ Same letters in parenthesis against data for each parameter indicates statistically insignificant difference $(p<0.05)$.

\begin{tabular}{|c|c|c|c|c|c|c|c|}
\hline riety & $\begin{array}{c}\text { Onset Temp }{ }^{\dagger} \\
\left({ }^{\circ} \mathrm{C}\right)\end{array}$ & Peak Temp ${ }^{\dagger}\left({ }^{\circ} \mathrm{C}\right)$ & $\begin{array}{c}\text { Peak Visco }^{\dagger} \\
\text { (Pa.s) }\end{array}$ & $\begin{array}{c}\text { Trough Visco }{ }^{\dagger} \\
\text { (Pa.s) }\end{array}$ & $\begin{array}{l}\text { Final Visco }{ }^{\dagger} \\
\text { (Pa.s) }\end{array}$ & $\begin{array}{c}\text { Breakdown } \\
\text { Visco }^{\dagger} \text { (Pa.s) }\end{array}$ & $\begin{array}{l}\text { Setback } \\
\text { viscosity }^{\dagger}\end{array}$ \\
\hline IC-564948 & $64.2 \pm 0.0(a)$ & $82.8 \pm 0.3(a)$ & $0.65 \pm 0.0(\mathrm{~lm})$ & $0.39 \pm 0.0(f g h)$ & $0.21 \pm 0.0(a)$ & $0.40 \pm 0.0(j)$ & $0.28 \pm 0.0(a)$ \\
\hline IC-564953 & $64.5 \pm 0.0(a)$ & $83.7 \pm 0.1(b)$ & $0.62 \pm 0.0(k)$ & $0.38 \pm 0.0(e f g)$ & $0.25 \pm 0.0(b)$ & $0.26 \pm 0.0(e f)$ & $0.28 \pm 0.0(a)$ \\
\hline IC-564950 & $65.94 \pm 0.0(b c)$ & $90.5 \pm 0.1(c)$ & $0.66 \pm 0.0(\mathrm{mn})$ & $0.36 \pm 0.0($ def $)$ & $0.28 \pm 0.0(d)$ & $0.30 \pm 0.0(g h)$ & $0.29 \pm 0.0(a b)$ \\
\hline IC-321204 & $65.6 \pm 0.0(b)$ & $90.3 \pm 0.0(c)$ & $0.677 \pm 0.0(o)$ & $0.40 \pm 0.0(g h)$ & $0.265 \pm 0.0(c)$ & $0.26 \pm 0.0(e f)$ & $0.30 \pm 0.0(b c)$ \\
\hline IC-558321 & $66.47 \pm 0.0(c d)$ & $91.35 \pm 0.0(d)$ & $0.67 \pm 0.0(n o)$ & $0.36 \pm 0.0($ def $)$ & $0.52 \pm 0.0(e)$ & $0.31 \pm 0.0(h i)$ & $0.30 \pm 0.0(b c)$ \\
\hline IC-321201 & $66.62 \pm 0.0(d)$ & $91.5 \pm 0.0(d)$ & $0.69 \pm 0.0(p)$ & $0.37 \pm 0.0($ def $)$ & $0.54 \pm 0.0(f)$ & $0.30 \pm 0.0(i)$ & $0.29 \pm 0.0(a b)$ \\
\hline IC-564939 & $66.0 \pm 0.6(c d)$ & $91.3 \pm 0.0(d)$ & $0.64 \pm 0.0(l)$ & $0.40 \pm 0.0(g h)$ & $0.82 \pm 0.0(j)$ & $0.20 \pm 0.0(d)$ & $0.52 \pm 0.0(f g)$ \\
\hline IC-332963 & $68.9 \pm 0.0(e)$ & $90.5 \pm 0.1(c)$ & $0.65 \pm 0.0(\mathrm{~m})$ & $0.49 \pm 0.0(i)$ & $1.00 \pm 0.0(p)$ & $0.14 \pm 0.0(b)$ & $0.54 \pm 0.0(g)$ \\
\hline IC-324133 & $70.3 \pm 0.0(f)$ & $90.5 \pm 0.1(c)$ & $0.62 \pm 0.0(\mathrm{k})$ & $0.51 \pm 0.0(i)$ & $0.56 \pm 0.0(g)$ & $0.12 \pm 0.0(a)$ & $0.30 \pm 0.0(b c)$ \\
\hline IC-321195 & $71.0 \pm 0.1(\mathrm{~g})$ & $90.3 \pm 0.1(c)$ & $0.62 \pm 0.0(k)$ & $0.51 \pm 0.0(i)$ & $0.60 \pm 0.0(h)$ & $0.29 \pm 0.0(g)$ & $0.31 \pm 0.0(c)$ \\
\hline IC-558311 & $72.8 \pm 0.0(h)$ & $91.1 \pm 0.0(d)$ & $0.57 \pm 0.0(i)$ & $0.34 \pm 0.0(c d)$ & $0.67 \pm 0.0(i)$ & $0.17 \pm 0.0(c)$ & $0.30 \pm 0.0(b c)$ \\
\hline IC-545288 & $73.2 \pm 0.0(h)$ & $92.3 \pm 0.0(e)$ & $0.53 \pm 0.0(h)$ & $0.39 \pm 0.0(f g h)$ & $0.68 \pm 0.0(i)$ & $0.21 \pm 0.0(d)$ & $0.31 \pm 0.0(c)$ \\
\hline IC-564949 & $74.3 \pm 0.0(i)$ & $92.3 \pm 0.0(f)$ & $0.43 \pm 0.0(\mathrm{~g})$ & $0.38 \pm 0.0(e f g)$ & $0.87 \pm 0.0(\mathrm{~m})$ & $0.25 \pm 0.0(e)$ & $0.36 \pm 0.0(d)$ \\
\hline IC-321206 & $74.5 \pm 0.0(j)$ & $92.5 \pm 0.0(g)$ & $0.41 \pm 0.0(f)$ & $0.25 \pm 0.0(a)$ & $0.90 \pm 0.0(o)$ & $0.17 \pm 0.0(c)$ & $0.37 \pm 0.0(d)$ \\
\hline IC-564952 & $75.5 \pm 0.5(j k)$ & $92.5 \pm 0.0(e)$ & $0.40 \pm 0.0(e f)$ & $0.39 \pm 0.0(f g h)$ & $0.88 \pm 0.0(n)$ & $0.18 \pm 0.0(c)$ & $0.46 \pm 0.0(e)$ \\
\hline IC-321193 & $75.53 \pm 0.5(k)$ & $93.3 \pm 0.0(\mathrm{~g})$ & $0.39 \pm 0.0(d e)$ & $0.30 \pm 0.0(b)$ & $0.855 \pm 0.0(k l)$ & $0.13 \pm 0.0(a)$ & $0.47 \pm 0.0(e)$ \\
\hline IC-564940 & $75.0 \pm 0.0(k)$ & $94.5 \pm 0.0(h)$ & $0.38 \pm 0.0(d)$ & $0.31 \pm 0.0(c)$ & $0.86 \mathrm{k} \pm 0.0(\mathrm{~lm})$ & $0.029 \pm 0.0(g)$ & $0.53 \pm 0.0(f g)$ \\
\hline IC-545274 & $76.1 \pm 0.1(l)$ & $93.3 \pm 0.1(g)$ & $0.36 \pm 0.0(c)$ & $0.35 \pm 0.0(d e)$ & $0.89 \pm 0.0($ no $)$ & $0.030 \pm 0.0(g h)$ & $0.52 \pm 0.0(f)$ \\
\hline IC-558315 & $78 \pm 0.0(m)$ & $95.3 \pm 0.0(i)$ & $0.27 \pm 0.0(b)$ & $0.31 \pm 0.0(b c)$ & $0.87 \pm 0.0(j k)$ & $0.031 \pm 0.0(i)$ & $0.53 \pm 0.0(g)$ \\
\hline IC-545197 & $78.78 \pm 0.1(n)$ & $94.7 \pm 0.0(j)$ & $0.25 \pm 0.0(a)$ & $0.35 \pm 0.0(d e)$ & $0.87 \pm 0.0(j k)$ & $0.027 \pm 0.0(f)$ & $0.56 \pm 0.0(h)$ \\
\hline
\end{tabular}


ing peak temperatures of starches from different cultivars of rice are in conformity with the temperature and viscosity pasting data reported for rice starches by Park et al. [15] and Li et al. [28]. The highest peak viscosity and lowest setback viscosity shown by starch pastes from the accession IC-583088, which is a waxy cultivar, are consistent with the fact that waxy starch comprises mainly of amylopectin which allows the granules to swell easily. Similar observations have been made by Tester and Morrison [19] and Morrison [46] for other waxy starches. Since setback viscosity reflects gel network formation after starch pasting [28], the near absence of amylose in "waxy" starch will result in fewer interactions among close-packed granules, resulting in low gel network formation. Thus, because of their greater resistance to thinning and shearing, starches from SKY-AK1608, IC-583035, YS-RC-219, IC-564939 and IC-332963 can be of importance to industry for various applications.

\section{Acknowledgements}

The study was supported by Grant No. BT/04/NE/2009 under the biotech Hub programme from Department of Biotechnology, Govt. of India, New Delhi (India).

\section{References}

[1] Shannon, J.C. and Garwood, D.L. (1984) Genetics and Physiology of Starch Development. In: Whistler, R.L., Be-Miller, J.N. and Paschell, E.F., Eds., Starch: Chemistry and Technology, Academic Press, Orlando, 25. http://dx.doi.org/10.1016/b978-0-12-746270-7.50009-4

[2] Makella, M.J. and Laakso, S. (2006) Studies on Oat Starch with a Celloscope: Granule Size and Distribution. Starch/Starke, 36, 159-163. http://dx.doi.org/10.1002/star.19840360503

[3] Evers, A.D. (1971) Scanning Electron Microscopy of Wheat Starch. III. Granule Development in the Endosperm. Starch/Starke, 23, 157. http://dx.doi.org/10.1002/star.19710230502

[4] Simmonds, D.H. and O’Brien, T.P. (1981) Morphological and Biochemical Development of Wheat Endosperm. Advanced Cereal Science Technology, 4, 5-14.

[5] Dengate, H. and Meredith, P. (1984) Variation in Size Distribution of Starch Granules from Wheat Grain. Journal of Cereal Science, 3, 83-90. http://dx.doi.org/10.1016/S0733-5210(84)80021-1

[6] Morrisson, W.R. and Scott, D.C. (1986) Measurements of the Dimensions of Wheat Starch Granule Populations Using a Coulter Counter with 100-Channel Analyser. Journal of Cereal Science, 4, 13-21. http://dx.doi.org/10.1016/S0733-5210(86)80003-0

[7] Lindebloom, N., Chang, P.R. and Tyler, R.T. (2004) Analytical, Biochemical and Hysiochemical Aspects of Starch Granule Size, with Emphasis on Small Granule Starches: A Review. Starch/Starke, 56, 89-99. http://dx.doi.org/10.1002/star.200300218

[8] Juliano, B.O. (1984) Rice Starch: Production, Properties and Uses. In: Whistler, R.L., BeMiller, J.N. and Paschall, E.F., Eds., Starch Chemistry and Technology, Academic Press, Orlando, 507-528. http://dx.doi.org/10.1016/b978-0-12-746270-7.50022-7

[9] Hoover, R., Smith, C., Zhou, Y. and Ratnayake, R.M.W.S. (2003) Physicochemical Properties of Canadian Oat Starches. Carbohydrate Polymers, 52, 253-261. http://dx.doi.org/10.1016/S0144-8617(02)00271-0

[10] Bao, J.S. and Bergman, C. (2004) The Functionality of Rice Starch. In: Eliasson, A.C., Ed., Starch in Food: Structure, Function and Applications, Woodhead Publishing, Cambridge, 152-184.

[11] Tester, R.F., Karkalas, J. and Qi, X. (2004) Starch Composition, Fine Structure and Architecture. Journal of Cereal Science, 39, 151-165. http://dx.doi.org/10.1016/i.jcs.2003.12.001

[12] Hizukuri, S. (1986) Polymodal Distribution of the Chain Lengths of Amylopectins and Its Significance. Carbohydrate Research, 147, 342-347. http://dx.doi.org/10.1016/S0008-6215(00)90643-8

[13] Wang, L.T., Bograchava, T.Y. and Hedley, C. (1998) Starch: As Simple as A, B, C: Review. Journal of Experimental Botany, 49, 480-502. http://dx.doi.org/10.1093/jxb/49.320.481

[14] Imberty, A., Buleon, A., Tran, V. and Perez, S. (1991) Recent Advances in Knowledge of Starch Structure. Starch/ Starke, 43, 375-384. http://dx.doi.org/10.1002/star.19910431002

[15] Park, I., Ibanez, A.M. and Shoemaker, C.F. (2007) Rice Starch Molecular Size and Its Relationship with Amylose Content. Starch/Starke, 59, 69-77. http://dx.doi.org/10.1002/star.200600568

[16] Patindol, J., Gu, X. and Wang, Y.-J. (2009) Chemometric Analysis of the Gelatinization and Pasting Properties of Long-Grain Rice Starches in Relation to Fine Structure. Starch/Starke, 63, 3-11. http://dx.doi.org/10.1002/star.200800022 
[17] Galliard, T. and Bowler, P. (1987) Morphology and Composition of Starch, In: Galliard, T., Ed. Starch: Properties and Potential, John Wiley \& Sons, New York, 281.

[18] Singh, N., Singh, J., Kaur, L., Sodhi, N.S. and Gill, B.S. (2003) Morphological, Thermal and Rheological Properties of Starches from Different Botanical Sources. Food Chemistry, 81, 219-231. http://dx.doi.org/10.1016/S0308-8146(02)00416-8

[19] Tester, R.F. and Morrison, W.R. (1990) Swelling and Gelatinization of Cereal Starches. 1. Effects of Amylopectins, Amylose, and Lipids. Cereal Chemistry, 67, 551-557.

[20] Graybosch, R.A. (1998) Waxy Wheats: Origin, Properties and Prospects. Trends in Food Science and Technology, 9 , 135-142. http://dx.doi.org/10.1016/S0924-2244(98)00034-X

[21] Bligh, F.J. (1999) Genetic Manipulation of Starch Biosynthesis: Progress and Potential. Biotechnology and Genetic Engineering Reviews, 16, 177-201. http://dx.doi.org/10.1080/02648725.1999.10647974

[22] Frei, M., Siddhuraju, P. and Becker, K. (2003) Studies on the in Vivo Starch Digestibility and the Glycemic Index of Six Different Indigenous Rice Cultivars from the Philippines. Food Chemistry, 83, 395-402. http://dx.doi.org/10.1016/S0308-8146(03)00101-8

[23] Mao, A.A., Hynniewta, T.M. and Sanjappa, M. (2009) Plant Wealth of Northeast India with Reference to Ethnobotany. Indian Journal of Traditional Knowledge, 8, 96-103.

[24] Takaoka, M., Wantanabe, S., Sassa, H., Yamamori, M., Nakamura, T., Sasakuma, T. and Hirano, H. (1997) Structural Characterization of High Molecular Weight Starch Granule-Bound Proteins in Wheat (Triticum aestivum L.). Journal of Agriculture and Food Chemistry, 45, 2929-2934. http://dx.doi.org/10.1021/jf9702656

[25] David, G.S., Russel, K.D., Jay-Lin, J. and George, E.I. (2006) Structures and Functional Properties of Starch from Seeds of Three Soyabean (Glycine max L. Merr.) Varieties. Starch/Starke, 58, 509-519. http://dx.doi.org/10.1002/star.200600534

[26] Juliano, B.O. (1971) A Simplified Assay for Milled Rice Amylose. Cereal Science Today, 16, 334-340.

[27] Thongbam, P.D., Raychaudhury, M., Durai, A., Das, S.P., Ramesh, T., Ramya, K.T., Fayaz, A.R. and Ngachan, S.V. (2012) Studies on Grain and Food Quality Traits of Some Indigenous Rice Cultivars of North-Eastern Hill Region of India. Journal of Agricultural Science, 4, 259-270.

[28] Li, Y., Shoemaker, C.F., Ma, T., Moon, T. and Zhong, F. (2008) Structure-Viscosity Relationships of Starches from Different Rice Varieties during Heating. Food Chemistry, 106, 1105-1115. http://dx.doi.org/10.1016/j.foodchem.2007.07.039

[29] Cruz, N.D. and Khush, G.S. (2000) Rice Grain Quality Evaluation Procedures. In: Singh, R.K., Singh, U.S. and Khush, G.S., Eds., Aromatic Rices, Oxford and IBH Publishing Co Pvt. Ltd, New Delhi, 15-28.

[30] Yeh, A.-I. and Li, J.-Y. (1996a) A Continuous Measurement of Swelling of Rice Starch during Heating. Journal of Cereal Science, 23, 277-283. http://dx.doi.org/10.1006/jers.1996.0028

[31] Li, J.-Y. and Yeh, A.-I. (2001) Relationships between Thermal, Rheological Characteristics and Swelling Power for Various Starches. Journal of Food Engineering, 50, 141-148. http://dx.doi.org/10.1016/S0260-8774(00)00236-3

[32] Zaidul, I.S.M., Yamauchi, H., Kim, S.J., Hashimoto, N. and Noda, T. (2007) RVA Study of Mixtures of Wheat Flour and Potato Starches with Different Phosphorus Content. Food Chemistry, 102, 1105-1111.

http://dx.doi.org/10.1016/j.foodchem.2006.06.056

[33] Puncha-Arnon, S., Puttanlek, C., Rungsardthong, V., Pathipanawat, W. and Uttapap, D. (2007) Changes in Physicochemical Properties and Morphology of Canna Starches during Rhizomal Development. Carbohydrate Polymers, 70, 206-217. http://dx.doi.org/10.1016/j.carbpol.2007.03.020

[34] Singh, S. and Singh, N. (2010) Relationship of Granule Size Distribution and Amylopectin Structure with Pasting, Thermal, and Retrogradation Properties in Wheat Starch. Journal of Agriculture and Food Chemistry, 58, 1180-1188. http://dx.doi.org/10.1021/jf902753f

[35] Manners, D.J. (1979) The Enzymatic Degradation of Starches. In: Blanshard, J.M.V. and Mitchell, J.R., Eds., Polysaccharides in Food, Butterworths, London, 5-91. http://dx.doi.org/10.1016/B978-0-408-10618-4.50010-X

[36] Wang, Y.-X., Ni, S., Chen, H.-Q., Liu, G.-F., Yang, J., Duan, B.-W. and Zhu, X.-D. (2010) Improvement of Method for Evaluating Amylose Content in Rice at Low Generations of Breeding. Chinese Journal of Rice Science, 24, 93-98.

[37] Dobo, M., Ayers, M., Walker, G. and Park, W.D. (2010) Polymorphism in the GBSS Gene Affects Amylose Content in US and European Rice Germplasm. Journal of Cereal Science, 52, 450-456. http://dx.doi.org/10.1016/j.jcs.2010.07.010

[38] Jimenez, R.R., Resurreccion, A.P. and Fitzgerald, M.A. (2010) Moving from Apparent to Actual Amylose in Rice. Proceedings of the 28th International Rice Research Conference, Hanoi, 8-12 November 2010, pp.

[39] Hoi, T.T., Nishi, A. and Satoh, H. (2008) Diversity of Granule Bound Starch Synthesis (GBSS) Levels in North Viet- 
nam Local Rice Cultivars. Rice Genetics Newsletter, 24, 62-64.

[40] Mutters, R.G. and Thomson, J.F. (2009) Rice Quality Handbook. University of California Agriculture and Natural Resources Pub. No. 3514.

[41] Lawal, O.S., Lapasin, R., Bellich, B., Olayiwola, T.O., Cesaro, A., Yoshimura, M. and Nishinari, K. (2011) Rheology and Functional Properties of Starches Isolated from Five Improved Rice Varieties from West Africa. Food Hydrocolloids, 25, 1785-1792. http://dx.doi.org/10.1016/j.foodhyd.2011.04.010

[42] Jayamani, P., Negrao, S., Brites, C. and Oliveira, M.M. (2007) Potential of Waxy Gene Microsatellite and Single-Nucleotide Polymorphisms to Develop Japonica Varieties with Desired Amylose Levels in Rice (Oryza sativa L.). Journal Cereal Science, 46, 178-186. http://dx.doi.org/10.1016/j.jcs.2007.03.003

[43] Rao, M.A., Okechukwu, P.E. and Silva, D.R. (1997) Rheological Behavior of Heated Starch Dispersions in Excess Water: Role of Starch Granule. Carbohydrate Polymers, 33, 273-283. http://dx.doi.org/10.1016/S0144-8617(97)00025-8

[44] Nurul, M.I., Azemi, B.M.N.M. and Manan, D.M.A. (1999) Rheological Behaviour of Sago (Metroxylon sagu) Starch Paste. Food Chemistry, 64, 501-505. http://dx.doi.org/10.1016/S0308-8146(98)00145-9

[45] Gibiński, M., Kowalski, S., Sady, M., Krawontka, J., Tomasik, P. and Sikora, M. (2006) Thickening of Sweet and Sour Sauces with Various Polysaccharide Combinations. Journal of Food Engineering, 75, 407-414. http://dx.doi.org/10.1016/j.jfoodeng.2005.04.054

[46] Morrison, W.R. (1995) Starch Lipids and How They Relate to Starch Granule Structure and Functionality. Cereal Foods World, 40, 437-446. 